\title{
Acknowledgement to Reviewers of Healthcare in 2017
}

\author{
Healthcare Editorial Office \\ MDPI AG, St. Alban-Anlage 66, 4052 Basel, Switzerland \\ Published: 10 January 2018
}

Peer review is an essential part in the publication process, ensuring that Healthcare maintains high quality standards for its published papers. In 2017, a total of 93 papers were published in the journal. Thanks to the cooperation of our reviewers, the median time to first decision was 26 days and the median time to publication was 54 days. The editors would like to express their sincere gratitude to the following reviewers for their time and dedication in 2017:

\begin{tabular}{|c|c|c|}
\hline Allen, Kachina & Guevara Lopez, Miguel Angel & Pérez-Calvo, Juan I. \\
\hline Allen, Herbert B & Gugusheff, Jessica & Peter, Richard \\
\hline Amiri, Azita & Guina, Jeffrey & Piazza, Jennifer \\
\hline An, Ruopeng & Gulis, Gabriel & Piccoli, GB \\
\hline Ansa, Benjamin & Gulis, Gabriel & Plateau, Carolyn \\
\hline Ansa, Benjamin & Hall, Jessica & Polanska, Kinga \\
\hline Ansa, Benjamin & Hammill, Bradley Gordon & Polimeni, Antonella \\
\hline Ansa, Benjamin & Harding, Mairead & Pollock, Richard L. \\
\hline Ansdell, Gary & Hargreaves, Dougal & Porrini, Esteban L. \\
\hline Antonanzas, Fernando & Harpin, Scott & Potvin, Noah \\
\hline Arnaert, Antonia & Harrington, Charles & Poulin, Patricia \\
\hline Assanelli, Deodato & Hawkins, Keely & Poutiainen, Ari \\
\hline Aurrekoetxea, Juan J. & Hayward, R. David & Pruitt, Sandi \\
\hline Aydede, Sema K & Heath, Gemma & Pryss, Rüdiger \\
\hline Bailey, Catherine & Hirsch, Annemarie G. & Quasney, Mike \\
\hline Basu, Pratyusha & Ho, Roger Chun-Man & Quigley, Eamon \\
\hline Bathula, Chandra & Howarth, Samuel J. & Rahman, Rahbel \\
\hline Bello, Aminu & Hung, Hsuan-Man & Ramsey, Vincent K. \\
\hline Benetou, Vassiliki & Hung, Hsuan-Man & Ratnamohan, Lux \\
\hline Bergbom, Ingegerd & Jacob, Jenna & Raven, Melissa \\
\hline Berkowsky, Ronald & Jacobsen, Ramune & Ray, Robin A \\
\hline Berkowsky, Ronald & Johansen, Guro Gravem & Reger, Michael \\
\hline Berliner, Janice L. & Jyväkorpi, Satu K & Reiter, E. Miranda \\
\hline Berry, Lois & Kabir, Thomas & Révész, Dóra \\
\hline Billiot, Shanondora & Kagan, Calvin M & Révész, Dóra \\
\hline Black, Helen K. & Kapellas, Kostas & Richmond, Janet \\
\hline Blankestijn, Peter J. & Kawano, Kouichiro & Rigby, Brandon Rhett \\
\hline Bodenheimer, Thomas & Keida, Elixabeth & Robinson, Ned \\
\hline Boisen, Kirsten A. & Kelley, Darshan & Rodrigues, Isabel \\
\hline Borovecki, Ana & Kim, Jung Yong & Roh, Young Sook \\
\hline
\end{tabular}




\begin{tabular}{|c|c|c|}
\hline Borry, Pascal & Kim, Kyung-su & Romeo, Giovanna \\
\hline Bowling, Christopher Barrett & Kim, Cheol-Ho & Ruetsch, Charles \\
\hline Brand, Serge & King, Irena B. & Rush, Elaine C \\
\hline Breitenfeld Granadeiro, Luiza & Knapp, Caprice A. & Russell, Joanna \\
\hline Brett-MacLean, Pamela & Koehler, Karsten & Sauder, Katherine Ann \\
\hline Brown-Wright, Lucy & Krams, Rob & Sawyer, Susan \\
\hline Bungum, Timothy & Krüger, Björn & Schalinski, Inga \\
\hline Caldeira, Silvia & Krysinska, Karolina & Schmid, Christopher \\
\hline Caldeira, Silvia & Kurokawa, Tetsuji & Schult, Tamara \\
\hline Camerino, Donatella & Kvaal, Kari & Shafiee, Mohammad A \\
\hline Camerino, Donatella & Lee, Paul H. & Shaw, Richard J. \\
\hline Caserta, Donatella & Leggat, Sandra G & Shaw, Margie Hodges \\
\hline Catherine, Hayes & LeMasters, Traci & Shepherd-Banigan, Megan \\
\hline Cayón De Las Cuevas, Joaquín & Lepp, Margret & Siega-Riz, Anna Maria \\
\hline Chang, Jer-Ming & Lesiuk, Teresa & Smith, Jared G \\
\hline Chang, Jer-Ming & Lewinska, Anna & Smith, Robert \\
\hline Chapple, Helen & Lewis, Joanne M. & Snaedal, Sunna \\
\hline Cheak-Zamora, Nancy & Lewis, Joanne M. & Sonestedt, Emily \\
\hline Christie, Deborah & Liabo, Kristin & Sotiriou, Elena \\
\hline Chu, Chun-Hung & Liapi, Charis & Soulage, Christophe \\
\hline Chu, Chun-Hung & Liapi, Charis & Spagnuolo, Rocco \\
\hline Chua, Tze-Ern & Lilja, Margareta & Spence, Marsha \\
\hline Chuang, Kai-Jen & Lin, Yi-Chin & Spilka, Jiří \\
\hline Clark, H. Westley & Loi, Natasha & Stansfeld, Stephen \\
\hline Clark, Stephanie Brown & Lophatananon, Artitaya & Stanton, Tasha \\
\hline Clifton, Andrew & Lophatananon, Artitaya & Stern, Tziporah \\
\hline Colver, Allan & Lu, Ying & Stins, John F. \\
\hline Comas, Maria & MacMahon, Kenneth & Stricker, Raphael \\
\hline Cooper, Sally-Ann & Macneill, Paul & Stroumpouki, Theodora \\
\hline Coronato, Antonio & Magnavita, Nicola & Stylianou, Yannis \\
\hline Coyne, Imelda & Magnavita, Nicola & Sudano, Laura Elizabeth \\
\hline Cusumano, Ana & Maisha, Buuma & Sukumar, Deeptha \\
\hline Danielson, Kirstie K. & Maltezou, Helen C. & Temple, Norman \\
\hline De Gruijl, Frank & Maltezou, Helen C. & Thomsen, Hauke \\
\hline De Perio, Marie & Manderscheid, Ronald W. & Thomsen, Hauke \\
\hline De Souza, Russell & Marcus, Esther-Lee & Tran, Binh Q. \\
\hline De Souza, Russell & Marlicz, Wojciech & Trevisan, Maurizio \\
\hline Denham, Bryan E. & Masanotti, Giuseppe & Trevisan, Andrea \\
\hline Denner, Larry & Mason, Deanna Marie & Trovik, Jone \\
\hline Dimitropoulos, Gina & Mason, Deanna Marie & Tso, For Yue \\
\hline Dominguez, Tyan Parker & Masso-Welch, Patricia A. & Turner, Gill \\
\hline Dontje, Manon & Masterson-Algar, Patricia & Van Ballegooijen, Hanne \\
\hline Dos Santos, Eduardo Ribeiro & Mathieu, Luc & Vecchio, Nerina \\
\hline
\end{tabular}




\begin{tabular}{|c|c|c|}
\hline Dryga, Anatoly & Mazur, Joanna & Vehkaoja, Antti \\
\hline Du, K.-L. & McConatha, Jasmin Tahmaseb & Venusia, Covelli \\
\hline Dumler, F & McDarby, Vincent & Venuti, Aldo \\
\hline Durbin, Janet & McKeown, Martin J. & Venuti, Aldo \\
\hline Efird, Jimmy & McNaughton, Nancy & Volpe, Stella \\
\hline El Ghoch, Marwan & Mearin, Fermin & Vuorio, Alpo \\
\hline Erazo, Marcia & Miller, Jennifer & Walker, Meghan J. \\
\hline Esposito, Ciro & Miller, Jessica & Wark, Stuart \\
\hline Evans, Mary & Mizuno, Koh & Wark, Stuart \\
\hline Fan, Jing & Mollica, Michelle & Watanabe, Eiichi \\
\hline Fauchier, Angele C & Molton, Ivan & Watson, Jacqueline \\
\hline Ferret, Rick & Mónica, Perez Bazán Laura & Weiner, Shira Schecter \\
\hline Firmino, Horacio & Morano, Kevin A. & Weiner, Shira Schecter \\
\hline Ford, Jon & Morano, Kevin A. & Wells, Yvonne \\
\hline Fourtounas, Costas & Morisset, Anne-Sophie & Wilkinson, Tom J. \\
\hline Fourtounas, Costas & Naseer, Noman & Wilson, Cristina \\
\hline Fourtounas, Costas & Neilson, Shane & Wiltshire, Esko J. \\
\hline Fratkin, Michael & Newman, John F. & Woo, Jessica Graus \\
\hline Freeman, Michael D. & Newman, John F. & Woodward, Alpha \\
\hline Friedman, Linda Weiser & Nikolaidis, Christos & Wu, Liyun \\
\hline Gaultney, Jane & Nikolaidis, Christos & Wu, Liyun \\
\hline Gaynor, Keith & Nishi, Nobuo & Wu, Liyun \\
\hline Gemert, Frederik Van & Nolan, Clodagh & Wu, Liyun \\
\hline Gerards, Sanne & Norman, Rachel & Wu, Ryanne Rebekah \\
\hline Gerdle, Björn & Nourazari, Sara & Wynia, Matthew \\
\hline Gitau, Mary & Nwanodi, Oroma & $\mathrm{Xu}$, Feng-Lian \\
\hline Gitlow, Lynn & O'Connell, Rhona & Yamamoto, Miwa \\
\hline Goldstein, Rachel Rosenberg & O’Grady, Lyn & Yao, YuChun \\
\hline Gomes, João Fernando Pereira & Olson, Michael W. & Yeh, May \\
\hline Goroll, Allan H. & Pagé, Gabrielle & You, Shingchern \\
\hline Goroll, Allan H. & Paludi, Michele & Zanutto, Alberto \\
\hline Gozal, Evelyne & Parsi, Kayhan & Zhang, Bing \\
\hline Gray, Andrew & Paul-Ward, Amy & Zhou, Shang-Ming \\
\hline Greaves, Lorraine & Peladic, Nikolina Jukic & Zong, Geng \\
\hline Guerrero-Mosquera, Carlos & Peled, Ronit & Zuchowski, Jessica L. \\
\hline
\end{tabular}

(C) 2018 by the authors. Licensee MDPI, Basel, Switzerland. This article is an open access article distributed under the terms and conditions of the Creative Commons Attribution (CC BY) license (http://creativecommons.org/licenses/by/4.0/). 